\title{
SIGNIFICADOS, ANÁLISIS CONCEPTUAL Y DISPOSICIÓN A PAGAR DE MEZCAL
} ANCESTRAL DE IXCATLÁN ${ }^{31}$

\author{
Susana López*, Anastacio Espejel, Arturo Hernández \\ Universidad Autónoma Chapingo: Km 38.5 Carretera México-Texcoco Chapingo, México. C.P. 56230 e- \\ mail: lopezgarciayareth@gmail.com
}

El mezcal ancestral es una bebida tradicional, obtenida de la destilación de jugos de agave fermentados y representativa de la cultura mexicana. El objetivo fue identificar los significados que representa el mezcal ancestral de Ixcatlán para consumidores locales; la conceptualización asociada y la disposición a pagar (DAP) un sobreprecio. Se aplicó una encuesta a 210 consumidores mediante elección libre donde se presentó una imagen a manera de estímulo del mezcal ancestral y se pidió escribieran tres palabras que evocaban. Las categorías de mayor comunalidad dentro del significado psicológico simbólico fueron tradición, proceso, identidad y hedonismo. Se obtuvo una lista de palabras mediante un procedimiento de libre asociación, se agruparon en categorías semánticas dentro de seis campos sociales, a las frecuencias se les aplicó una prueba de k proporciones. La conceptualización de mezcal ancestral incluyó atributos tangibles e intangibles; los consumidores de Oaxaca lo asociaron con tradición, identidad, calidad y atributos sensoriales y hedonismo. Se realizaron regresiones logísticas para obtener las variables de mayor influencia en la disposición a pagar por mezcales ancestrales. El mezcal ancestral elaborado por pequeños productores presentó la mayor disposición a pagar (90.95\%).

Palabras clave: mezcal ancestral, comunalidad, conceptualización, disposición a pagar.

\section{Introducción}

Un alimento tradicional es un producto elaborado de forma específica, acorde a la herencia gastronómica, con características sensoriales particulares, vinculado a una determinada área local, región o país [véase Guerrero et al. (2009)]. El mezcal es una bebida tradicional producida por la destilación de jugo de agave fermentado [véase Vera-Guzmán et al. (2010)]; de acuerdo a la NOM-070-SCFI-2016 se tienen tres categorías de acuerdo al proceso y materiales utilizados durante su producción. En la valorización de los productos el significado forma parte de la elección del consumidor y en la valoración los consumidores asignan el valor económico a un producto diferenciado [véase Bakar et al. (2013); Barrera-Rodríguez et al. (2019)]. Por ello, los objetivos de esta investigación fueron: identificar comunalidades de categorías de significados en consumidores locales de mezcal ancestral de Ixcatlán; explorar la conceptualización asociada al mezcal ancestral en consumidores de seis zonas de México; e identificar los factores que influyen en la DAP un sobreprecio por mezcales ancestrales.

\section{Metodología}

\subsection{Significados}

Se aplicó una encuesta de elección libre a una muestra de consumidores locales $(n=210)$ de Santa María Ixcatlán Oaxaca, conformada por personas mayores de 40 años de ambos géneros. A cada uno de los consumidores se le mostró una imagen de mezcal ancestral y se les pidió que escribieran las tres palabras o ideas que evocaran con relación al estímulo presentado [véase Elliot (1994)]. Las respuestas fueron agrupadas en categorías de significados y a las frecuencias de palabras asociadas a cada una de las categorías se les aplicó una prueba de k proporciones y procedimiento de Marascuilo.

\subsection{Análisis conceptual y disposición a pagar}

Se aplicó una encuesta apoyándose de la aplicación Formularios Google a 210 consumidores de mezcal mayores de 18 años. En la primera sección de la encuesta se preguntó a los consumidores, aspectos sociodemográficos (género, edad, estado de procedencia, escolaridad, ocupación e ingreso mensual). En la segunda sección, se presentaron las palabras "mezcal ancestral" y se solicitó al consumidor que escribiera tres palabras o términos que asociaran. La tercera sección constó de cuatro aseveraciones asociadas a la decisión de compra de mezcal ancestral: 1) diseño de envase y etiquetado;2) certificación; 3) accesibilidad de mercado; y 4) conocimiento adquirido sobre el mezcal y experiencias relacionadas con él. En la cuarta sección se presentaron siete atributos tangibles (sabor, aroma, color, calidad, envase, etiqueta y grados de alcohol). En la sección cinco se solicitó indicaran que tan importante es el precio, la producción local o regional, producción tradicional, accesibilidad en el mercado y la destilación en olla de barro. La evaluación se hizo empleando escalas likert. En la sección seis se preguntó la DAP por: 1) mezcal ancestral; 2) mezcal

${ }^{31}$ Financiado con fondos del Proyecto 21004-DTT-62 de la DGIP-Universidad Autónoma Chapingo 
ancestral producido con agaves silvestres; 3) mezcal ancestral producido por pequeños productores; y 4) mezcal ancestral que contará con Indicación Geográfica; empleando una escala binaria (1“dispuesto a pagar"; 0“sin disposición a pagar") y el porcentaje de sobreprecio a pagar (5\%, 10\%, 15\%, 20\%, 25\% y $>25 \%$ ). Se obtuvieron categorías y proporciones de las conceptualizaciones. Para describir la disposición a pagar un sobreprecio se realizaron regresiones logísticas a los datos obtenidos mediante un modelo Logit, estimado por máxima verosimilitud [véase Loureiro \& Hine (2002)].

\section{Resultados}

\subsection{Significados del mezcal ancestral de Ixcatlán}

Las diferentes ideas o palabras empleadas por los consumidores locales fueron organizadas en 18 categorías de significados para el mezcal ancestral de Ixcatlán; ocho categorías (añoranza familiar y social, creencias religiosas, emociones y sentimientos, hedonismo, identidad, proceso, tipicidad y tradición) se agruparon dentro del significado psicológico; las diez restantes (atributo de aroma, atributos de sabor, bebida y accesorios, calidad, economía, efecto negativo, materia prima, salud, turismo, usos y beneficios) se agruparon en el significado psicológico utilitario. Dentro de los significados psicológicos, el estadístico de prueba $\mathrm{X}^{2}$ resultó significativo $(\mathrm{p}<0.05)$, lo que indicó diferencia de proporciones entre las comunalidades de los significados utilitarios y simbólicos. En relación al estadístico de prueba $\mathrm{X}^{2}$ para el análisis de $\mathrm{k}$ proporciones para las diversas categorías de significados del mezcal ancestral de Ixcatlán de igual manera resultó significativo $(\mathrm{p}<0.05)$ para el global de consumidores. Las categorías de significados que presentaron las mayores comunalidades fueron: tradición, proceso, identidad, hedonismo y bebidas y accesorios (Gráfico 1).

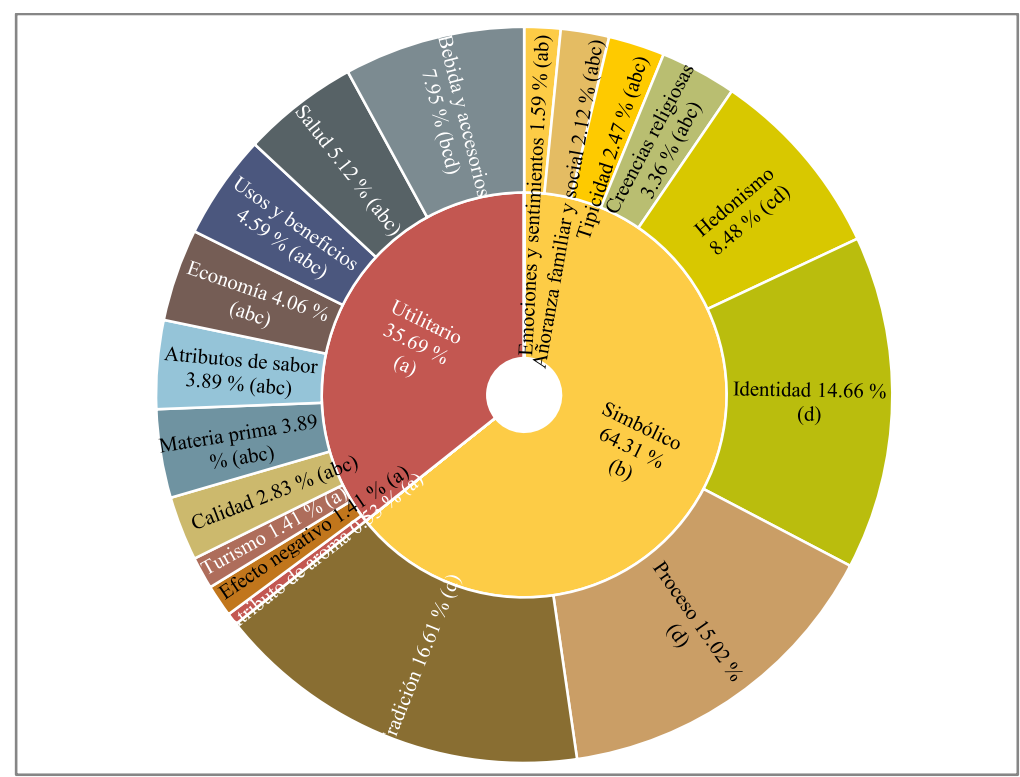

Gráfico 4. Comparaciones de los porcentajes de las comunalidades de las categorías de significados otorgados al mezcal ancestral de Ixcatlán y sus aportaciones a los significados psicológicos.

\subsection{Análisis conceptual}

En el Cuadro 1 se muestran los porcentajes de las categorías semánticas y la comparación de estas dentro de cada campo social. El estadístico de prueba de $\mathrm{X}^{2}$ para el análisis de $\mathrm{k}$ proporciones para las diversas categorías semánticas por campos sociales resultó significativo $(\mathrm{p}<0.05)$ para los campos sociales de Oaxaca y Estado de México. Las categorías en Oaxaca se clasificaron en tres grupos (a,b,c), de los cuales el grupo c presentó los mayores porcentajes integrado por tradición, identidad, calidad y atributos sensoriales y hedonismo; en el Estado de México también se obtuvieron tres grupos de categorías (a,b,c) y el grupo con los mayores porcentajes estuvo integrado por tradición, identidad, calidad y atributos sensoriales, hedonismo, proceso y tipicidad. Por otra parte, en Michoacán, Puebla, Durango y Ciudad de México las categorías resultaron estadísticamente iguales ( $\mathrm{p}<0.05)$.

Cuadro 1. Porcentajes de las categorías para mezcal ancestral por campo social

\begin{tabular}{llccccl}
\hline Categoría & Michoacán & Puebla & Durango & Oaxaca & $\begin{array}{l}\text { Ciudad } \\
\text { de } \\
\text { México }\end{array}$ & $\begin{array}{l}\text { Estado de } \\
\text { México }\end{array}$ \\
\hline Tradición & $28.57 \mathrm{a}^{z}$ & $26.67 \mathrm{a}$ & $21.95 \mathrm{a}$ & $24.69 \mathrm{c}$ & $29.55 \mathrm{a}$ & $22.35 \mathrm{c}$ \\
Identidad & $14.29 \mathrm{a}$ & $13.33 \mathrm{a}$ & $9.76 \mathrm{a}$ & $13.99 \mathrm{bc}$ & $18.18 \mathrm{a}$ & $15.29 \mathrm{abc}$ \\
\hline
\end{tabular}




\begin{tabular}{lllllll}
\hline Calidad y atributos & $11.9 \mathrm{a}$ & $17.78 \mathrm{a}$ & $24.39 \mathrm{a}$ & $12.76 \mathrm{bc}$ & $11.36 \mathrm{a}$ & $18.82 \mathrm{bc}$ \\
sensoriales & $9.52 \mathrm{a}$ & $11.11 \mathrm{a}$ & $9.76 \mathrm{a}$ & $12.35 \mathrm{bc}$ & $6.82 \mathrm{a}$ & $12.94 \mathrm{abc}$ \\
Hedonismo & $7.14 \mathrm{a}$ & $4.44 \mathrm{a}$ & $4.88 \mathrm{a}$ & $9.47 \mathrm{ab}$ & $4.55 \mathrm{a}$ & $7.65 \mathrm{abc}$ \\
Proceso & $7.14 \mathrm{a}$ & $8.89 \mathrm{a}$ & $12.2 \mathrm{a}$ & $8.23 \mathrm{ab}$ & $9.09 \mathrm{a}$ & $4.71 \mathrm{a}$ \\
Bebida y accesorios & $4.76 \mathrm{a}$ & $8.89 \mathrm{a}$ & $4.88 \mathrm{a}$ & $7.82 \mathrm{ab}$ & $6.82 \mathrm{a}$ & $8.82 \mathrm{abc}$ \\
Tipicidad & $9.52 \mathrm{a}$ & $4.44 \mathrm{a}$ & $7.32 \mathrm{a}$ & $7.82 \mathrm{ab}$ & $6.82 \mathrm{a}$ & $5.29 \mathrm{ab}$ \\
Salud & $7.14 \mathrm{a}$ & $4.44 \mathrm{a}$ & $4.88 \mathrm{a}$ & $2.88 \mathrm{a}$ & $6.82 \mathrm{a}$ & $4.12 \mathrm{a}$ \\
Materia prima &
\end{tabular}

${ }^{2}$ Proporciones con distinta letra en columnas, son estadísticamente diferentes $(\mathrm{p}<0.05)$.

\subsection{Disposición a pagar un sobreprecio por mezcal ancestral}

En el Cuadro 2 se muestra la significancia de las regresiones logísticas de la DAP para cada tipo de mezcal ancestral en función a las características sociodemográficas, aseveraciones de compra, atributos tangibles e importancia de las características del producto. Con relación a las características sociodemográficas resultaron significativas $(\mathrm{p}<0.05)$ asociadas a la DAP por $750 \mathrm{~mL}$ de cada tipo de mezcal ancestral, caso contrario, para las aseveraciones de compra, excepto para el mezcal ancestral si contara con Indicación Geográfica. Las regresiones logísticas de la DAP con atributos tangibles resultaron significativas para mezcal ancestral, y de agaves silvestres; y las regresiones logísticas de la DAP con las características del producto también resultaron significativas, a excepción del mezcal ancestral si contara con Indicación Geográfica. La DAP un sobreprecio por los diferentes tipos de mezcal ancestral ejerció influencia significativa por el ingreso, certificaciones, calidad, sabor y destilación en olla de barro.

Cuadro 2. Significancia de las regresiones logísticas de las variables que influyen en la disposición a pagar (DAP) por el método de máxima verosimilitud.

\begin{tabular}{lcccc}
\hline \multirow{2}{*}{ Tipo de mezcal ancestral } & \multicolumn{4}{c}{ Variables } \\
\cline { 2 - 5 } & $\begin{array}{c}\text { Características } \\
\text { sociodemográficas }\end{array}$ & $\begin{array}{c}\text { Aseveraciones } \\
\text { de compra }\end{array}$ & $\begin{array}{c}\text { Atributos } \\
\text { tangibles }\end{array}$ & $\begin{array}{c}\text { Características } \\
\text { del producto }\end{array}$ \\
\hline Mezcal ancestral & $<\mathbf{0 . 0 0 0 1}$ & 0.725 & $\mathbf{0 . 0 0 2}$ & $\mathbf{0 . 0 3 0}$ \\
$\begin{array}{l}\text { Mezcal de agaves silvestres } \\
\text { Mezcal producido por pequeños }\end{array}$ & $<\mathbf{0 . 0 0 0 1}$ & 0.467 & $\mathbf{0 . 0 0 4}$ & $\mathbf{0 . 0 0 1}$ \\
$\begin{array}{l}\text { productores } \\
\text { Mezcal con Indicación }\end{array}$ & $<\mathbf{0 . 0 0 0 1}$ & 0.628 & 0.101 & $\mathbf{0 . 0 1 6}$ \\
Geográfica & $<\mathbf{0 . 0 0 0 1}$ & $\mathbf{0 . 0 3 8}$ & 0.098 & 0.164 \\
\hline
\end{tabular}

La DAP por los cuatro tipos de mezcal ancestral (Cuadro 3), muestra que el $18.57 \%$ de consumidores no mostró DAP por un mezcal ancestral si contara con IG, estadísticamente igual a un mezcal ancestral elaborado de agaves silvestres y mezcal ancestral, mientras que para mezcal ancestral elaborado por pequeños productores solo el $9.05 \%$ y el resto $(90.95 \%)$ si reveló DAP. La disposición a pagar un sobreprecio de $10 \%, 15 \%, 20 \%$ y $25 \%$ no mostró diferencia significativa entre los cuatro tipos de mezcal, destacando que, para el $10 \%$ existe un mayor número de consumidores, lo que puede asociarse a un porcentaje más accesible para la mayoría de los consumidores. Además, se puede observar que el 14.76\% de consumidores está dispuesto a pagar un sobreprecio mayor a 25\% para mezcal ancestral elaborado a partir de pequeños productores, contrario a un mezcal ancestral con distintivo de IG, con solo un 5.24\%.

Cuadro 3. Disposición a pagar (\%) un sobreprecio en consumidores por tipo de mezcal ancestral.

\begin{tabular}{lccccccc}
\hline \multicolumn{1}{c}{$\begin{array}{c}\text { Tipo de mezcal } \\
\text { ancestral }\end{array}$} & $0 \%$ & $5 \%$ & $10 \%$ & $15 \%$ & $20 \%$ & $25 \%$ & $>25 \%$ \\
\cline { 2 - 7 } & $12.38 \mathrm{ab}^{\mathrm{z}}$ & $17.62 \mathrm{ab}$ & $25.71 \mathrm{a}$ & $14.76 \mathrm{a}$ & $15.24 \mathrm{a}$ & $6.67 \mathrm{a}$ & $7.62 \mathrm{ab}$ \\
$\begin{array}{l}\text { Mezcal ancestral } \\
\text { Mezcal de agaves } \\
\text { silvestres }\end{array}$ & $12.38 \mathrm{ab}$ & $21.90 \mathrm{ab}$ & $20.48 \mathrm{a}$ & $16.19 \mathrm{a}$ & $13.81 \mathrm{a}$ & $7.62 \mathrm{a}$ & $7.62 \mathrm{ab}$ \\
$\begin{array}{l}\text { Mezcal producido } \\
\text { por pequeños } \\
\text { productores }\end{array}$ & $9.05 \mathrm{a}$ & $14.76 \mathrm{a}$ & $21.90 \mathrm{a}$ & $16.67 \mathrm{a}$ & $12.86 \mathrm{a}$ & $10.00 \mathrm{a}$ & $14.76 \mathrm{~b}$ \\
$\begin{array}{l}\text { Mezcal con } \\
\begin{array}{l}\text { Indicación } \\
\text { Geográfica }\end{array}\end{array}$ & & & & & & \\
\hline
\end{tabular}

\section{Conclusiones}

Las categorías de mayor comunalidad dentro del significado psicológico simbólico fueron tradición, proceso, identidad y hedonismo. La conceptualización de mezcal ancestral incluyó atributos tangibles e 
intangibles. Oaxaca lo asoció mayormente con tradición, identidad, calidad y atributos sensoriales y hedonismo. La DAP un sobreprecio por mezcal ancestral ejerció influencia significativa por la calidad, sabor, destilación en olla de barro, certificaciones y el ingreso. El mezcal ancestral elaborado por pequeños productores presentó la mayor disposición a pagar $(90.95 \%)$.

\section{Bibliografía}

Bakar, A., Lee, R., \& Rungie, C. (2013). The effects of religious symbols in product packaging on Muslim consumer responses. Australasian Marketing Journal (AMJ), 21(3):198-204.

Barrera-Rodríguez, A. I., Cuevas-Reyes, V., \& Espejel-García, A. (2019). Factores de valoración en consumidores de mezcal en Oaxaca. Estudios sociales. Revista de alimentación contemporánea y desarrollo regional, 29(54).

Elliot, R. (1994). Exploring the symbolic meaning of brands. British Journal of Management, 5:13-19.

Guerrero, L., Claret, A., Verbeke, W., Enderli, G., Zakowska-Biemans, S., Vanhonacker, F., Issanchou, S., Sajdakowska, M., Granli, B. S., Scalvedi, L., Contel, M., \& Hersleth, M. (2010). Perception of traditional food products in six European regions using free word association. Food Quality and Preference, 21(2):225-233.

Vera-Guzmán, A. M., Guzmán-Gerónimo, R. I., \& López, M. G. (2010). Major and minor compounds in a mexican spirit, young mezcal coming from two agave species. Czech J. Food Sci, 28(2), 127-132.

Loureiro, M. L., \& Hine, S. E. (2002). Discovering Niche Markets: a comparison of consumer willingness to pay for local (Colorado Grown), Organic, and GMO-Free products. Journal of Agricultural and Applied Economics, 34(3):77-487. 